Physics, Chemistry, and Dynamics of Interplanetary Dust

ASP Conference Series, Vol. 104, 1996

Bo A. S. Gustafson and Martha S. Hanner (eds.)

\title{
Fragmentation of Comet Shoemaker-Levy 9's Nuclei During Flight Through the Jovian Atmosphere
}

\author{
Z. Sekanina \\ Jet Propulsion Laboratory, California Institute of Technology, \\ Pasadena, CA 91109
}

\begin{abstract}
Fragmentation dominates the ablation process of cometary type impactors during their atmospheric flight. Models that account for it imply that a kilometer-sized nuclear fragment of comet Shoemaker-Levy 9 has disintegrated almost completely by the time of its terminal explosion, at a pressure level of $<1$ bar. This result, consistent with impact scenarios based on precursor-event timings, suggests that the initial masses of two fragments, $K$ and $R$, were between $7 \times 10^{14}$ and $5 \times 10^{15}$ grams apiece.
\end{abstract}

Impact observations of the fragments $\mathrm{K}$ and $\mathrm{R}$ of comet Shoemaker-Levy 9 made, respectively, on July 19 and 21, 1994 from the ground and onboard the Galileo spacecraft are combined to constrain the penetration depths and the bulk properties of the impactors. The approach applied is based on slightly modified equations used in, and on ablation rates derived from, investigations of massive bolides in the Earth's atmosphere.

For "soft", cornetary impactors these studies indicate that fragmentationboth continuous and occurring in discrete events that last $\leq 0.1 \mathrm{~s}$-is the ablation process of paramount importance, vastly surpassing the effects of evaporation and spraying. The susceptibility to vigorous fragmentation is independent of the impactor's mass, with the necessary consequence of a precipitous loss of mass during atmospheric flight, an effect that has been neglected or grossly underestimated in most impact models for Shoemaker-Levy 9 proposed to date.

Meteor physicists have long been aware of the significance of fragmentation. Recently, its role was illustrated convincingly on the bolide Śumava (EN 041274) by Borovička \& Spurný (1995), who showed that this morphological analogue to comet Shoemaker-Levy 9 and the most massive cometary bolide on record had an initial mass of $\sim 5$ tons, a bulk density $0.1 \mathrm{~g} / \mathrm{cm}^{3}$, and an effective ablation coefficient of $0.32 \mathrm{~s}^{2} / \mathrm{km}^{2}$. Its luminous trail began at $99 \mathrm{~km}$ above sea level, equivalent to $\sim 380 \mathrm{~km}$ above 1 bar in the Jovian atmosphere, and the object disintegrated entirely at an altitude of $59 \mathrm{~km}$, equivalent to $\sim 190 \mathrm{~km}$ on Jupiter, at a dynamic pressure of $\sim 1$ bar and an atmospheric pressure of 0.25 mbar.

It is also known that large impactors explode instantly (e.g., McCrosky et al 1971, Sekanina 1983) at the terminal point of the luminous trajectory where the encountered atmospheric resistance can no longer be tolerated, i.e., at the point of peak dynamic pressure. In a simple model for a point source explosion, adapted to the case of an exponential atmosphere by Kompaneets (1960), a strong shock begins to propagate from the explosion point, with all the mass concentrated in a thin shell at its front (the shock regime). The pressure behind the front is proportional to the explosion's total energy and inversely to 
the expanding cavity's volume. The shock continues to expand at an accelerated rate upwards. The recondensed debris gradually trails behind (Boslough et al. 1994) and, because of Jupiter's gravity, it eventually follows a ballistic trajectory (the ballistic regime). The ejecta's maximum elevation was observed to be nearly independent of the explosion energy (Hammel et al.1995), implying an equivalent "initial" upward velocity of $\sim 13 \mathrm{~km} / \mathrm{s}$. Modelling this evolution constrains the time span between the explosion and the plume's emersion at the Jovian limb, the primary contributor to the observed timing of the precursor events, $t_{\wedge}-t_{V}$ (Figure 1). Similar conceptual models have independently been proposed by others (e.g., Boslough et al. 1995, Graham et al. 1995, Nicholson et al. 1995a).

The nominal scenarios for the nuclei $K$ and $R$, listed in Table 1 , are based on two assumed ablation coefficients, on the observed times $t_{\wedge}-t_{V}$ of $53 \mathrm{~s}$ for $\mathrm{K}$ (interpolated from the data by Watanabe et al. 1995) and $54 \mathrm{~s}$ for R (Graham et al. 1995), and on the effective initial diameters of $3.2 \mathrm{~km}$ for $\mathrm{K}$ (Sekanina, unpublished) and $2.4 \mathrm{~km}$ for R (Sekanina 1995), as determined photometrically from Hubble Space Telescope (HST) images. The impactors' initial masses are found to have ranged from $8 \times 10^{14}$ to $5 \times 10^{15} \mathrm{~g}$, their bulk densities from 0.1 to $0.3 \mathrm{~g} / \mathrm{cm}^{3}$, and their residual masses from $2 \times 10^{12}$ to $7 \times 10^{12} \mathrm{~g}$, or less than $1 \%$ of their preatmospheric masses. The explosion energies were near $10^{26} \mathrm{ergs}$ and the explosion altitudes 40 to $60 \mathrm{~km}$ above 1 bar. The shock's downward penetration (Kompaneets 1960) should have extended for at least $30 \mathrm{~km}$, thus affecting the $\mathrm{NH}_{3}$ clouds. If the plumes were products of line sources (Boslough et al. 1994), the tabulated residual mass would be a lower limit to the plume's actual mass.

Table 1. Nominal ablation scenarios for fragments $\mathrm{K}$ and $\mathrm{R}$

\begin{tabular}{|c|c|c|c|c|}
\hline \multirow[b]{2}{*}{ Ablation coefficient $\left(\mathrm{s}^{2} / \mathrm{km}^{2}\right)$ : } & \multicolumn{2}{|c|}{ Fragment $\mathrm{K}$} & \multicolumn{2}{|c|}{ Fragment $\mathrm{R}$} \\
\hline & 0.2 & 0.4 & 0.2 & 0.4 \\
\hline \multicolumn{5}{|l|}{ IMPACTOR AT TIME OF ATMOSPHERIC ENTRY } \\
\hline Preatmospheric mass $(\dot{\mathrm{g}})$ & $10^{15.27}$ & $10^{15.69}$ & $10^{14.88}$ & $10^{15.34}$ \\
\hline Kinetic energy (erg) ${ }^{a}$ & $10^{28.51}$ & $10^{28.93}$ & $10^{28.12}$ & $10^{28.58}$ \\
\hline Bulk density $\left(\mathrm{g} / \mathrm{cm}^{3}\right)$ & 0.11 & 0.29 & 0.10 & 0.30 \\
\hline Effective diameter (km) & 3.2 & 3.2 & 2.4 & 2.4 \\
\hline \multicolumn{5}{|l|}{ IMPACTOR AT TIME OF TERMINAL EXPLOSION } \\
\hline Residual mass $(\mathrm{g})$ & $10^{12.74}$ & $10^{12.86}$ & $10^{12.34}$ & $10^{12.50}$ \\
\hline Residual energy (erg) & $10^{25.98}$ & $10^{26.10}$ & $10^{25.58}$ & $10^{25.74}$ \\
\hline Residual effective diameter (km) & 0.46 & 0.36 & 0.34 & 0.27 \\
\hline Altitude above 1 bar $(\mathrm{km})$ & 50 & 44 & 57 & 49 \\
\hline Ambient atmospheric pressure (bar) & 0.080 & 0.11 & 0.058 & 0.085 \\
\hline Aerodynamic pressure (bar) & 562 & 795 & 383 & 600 \\
\hline Preatmospheric-to-residual mass ratio & 340 & 680 & 350 & 700 \\
\hline \multicolumn{5}{|l|}{ Time Line of Events Behind Jovian Limb $b$} \\
\hline Impactor's flight from disappearance to explosion (s) & 7.9 & 8.0 & 4.4 & 4.5 \\
\hline Plume's expansion in shock regime $(\mathrm{s})$ & 38.0 & 37.2 & 49.6 & 49.5 \\
\hline Plume's expansion in ballistic regime (s) & 7.1 & 7.8 & 0.0 & 0.0 \\
\hline Total time behind Jovian limb $b^{c}(s)$ & 53.0 & 53.0 & 54.0 & 54.0 \\
\hline
\end{tabular}




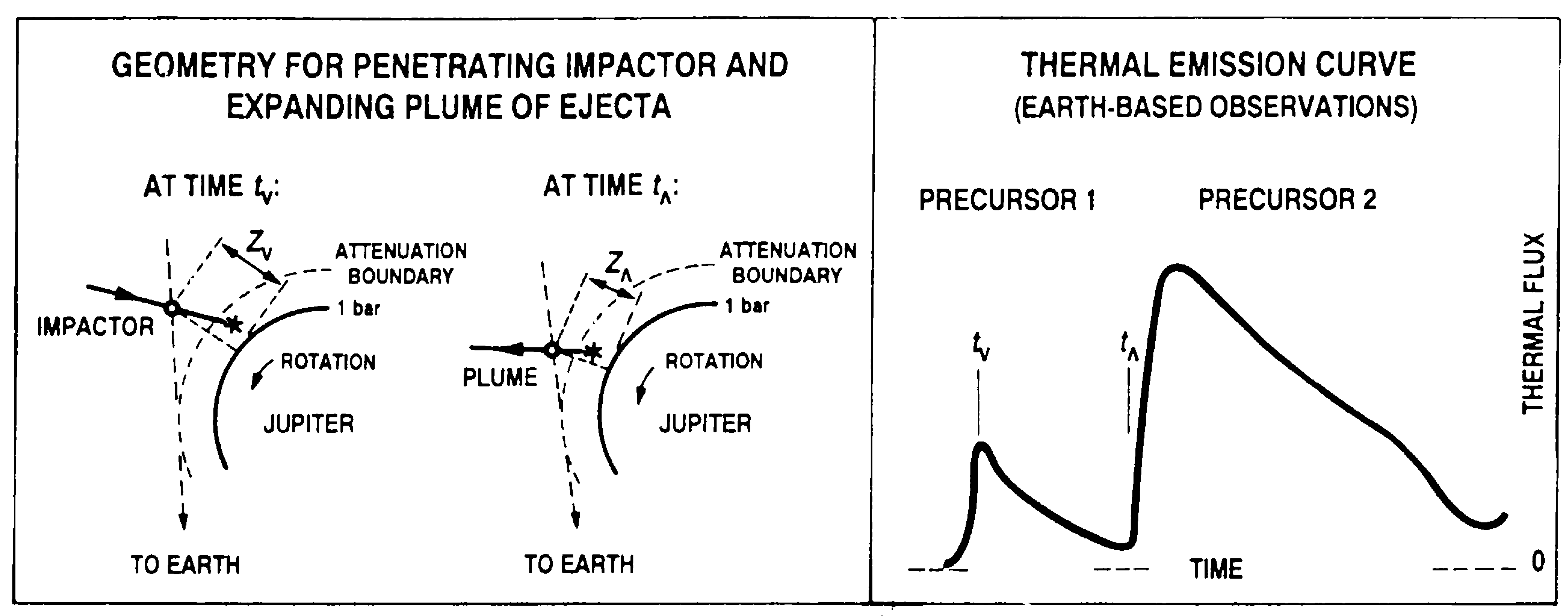

Figure 1. Geometry for a massive impactor penetrating the Jovian atmosphere and for an expanding plume of hypervelocity ejecta (left panel); and the pre-main event portion of the thermal emission curve as observed from the ground (right panel). The flux increases until the impactor disappears behind the limb at altitude $Z_{V}$ at time $t_{V}$, when Precursor 1 reaches its peak. The signal then subsides, being due to the wake of ablated material and a trailing column of the disturbed atmosphere. The terminal explosion of the impactor's residual mass is hidden behind the limb. The sudden onset of Precursor 2, at time $t_{\wedge}$, coincides with the front of the expanding ejecta's plume as it emerges over the $\operatorname{limb}$ at altitude $Z_{\Lambda}$. The difference $t_{\Lambda}-t_{\vee}$, the total time of the events occurring behind the limb, is the impact's key parameter.

The results in Table 1 are consistent with the timing of relevant events from experiments carried out onboard the Galileo spacecraft. The explosion time for $\mathrm{K}, 10: 24: 17 \mathrm{UT}$, coincides with the sharp peak on the light curve (Chapman et al. 1995) to $1 \mathrm{~s}$, whereas for $\mathrm{R}$ the explosion time, 05:34:57 UT, differs from Carlson et al.'s (1995) reference time by $11 \mathrm{~s}$, within their errors of measurement.

The $2.3 \mu \mathrm{m}$ fluxes reported for Precursors 1 by Watanabe et al. (1995) for $\mathrm{K}$ and by Graham et al. (1995) for R were used to derive the impactors' effective surface areas, $S_{\text {eff }}$, in units of their initial frontal areas $A_{0}$, and the product of a heat transfer coefficient $\Lambda<1$ and a fraction $\chi<1$ of the total energy spent on thermal reradiation as functions of temperature. The results, in Table 2, show that the signal from $R$ at $530 \mathrm{~km}$ constrains the effective temperature at that altitude to $\leq 1000 \mathrm{~K}$. Higher temperatures could, however, apply at the two lower altitudes. Nicholson et al. (1995b) found a color temperature of $1000 \mathrm{~K}$. In the Earth's atmosphere, the surface temperature range for a $60 \mathrm{~km} / \mathrm{s}$ projectile at equivalent altitudes has been estimated at $\sim 1500$ to $\sim 3000 \mathrm{~K}$ (Bronshten 1983).

The possibility of a complete or nearly complete disintegration of impactors before their terminal explosion, predicted from the present model, is consistent with the reported failure, by a number of observers, to detect the ejecta plumes for virtually all of the off-train fragments, such as $B, F, P_{2}$, or $V$. Since the residual mass of each impactor depends on its initial mass, velocity, and ablation (fragmentation) rate, it is apparent that the off-train nuclei were both smaller and less cohesive than the on-train nuclei, fragmenting almost spontaneously. 
Table 2. Effective temperature $T_{\text {eff }}$, effective emitting area $S_{\text {eff }}$ (in units of impactor's preatmospheric frontal area $A_{0}$ ), and a product $\dot{\chi} \Lambda$ from observed thermal fluxes for Precursors 1 of fragments $R$ and $K$

\begin{tabular}{|c|c|c|c|c|c|c|}
\hline \multirow{2}{*}{$\begin{array}{c}\text { Effective } \\
\text { temperature } \\
T_{\text {eff }}(\mathrm{K})\end{array}$} & \multicolumn{2}{|c|}{$\begin{array}{l}\text { Fragment } R \text { at } \\
\text { altitude of } 245 \mathrm{~km}\end{array}$} & \multicolumn{2}{|c|}{$\begin{array}{c}\text { Fragment } R \text { at } \\
\text { altitude of } 530 \mathrm{~km}\end{array}$} & \multicolumn{2}{|c|}{$\begin{array}{c}\text { Fragment } \mathrm{K} \text { at } \\
\text { altitude of } 390 \mathrm{~km}\end{array}$} \\
\hline & $S_{\text {eff }} / A_{0}$ & $x \Lambda$ & $S_{\text {eff }} / A_{0}$ & $x \Lambda$ & $S_{\text {eff }} / A_{0}$ & $x \Lambda$ \\
\hline 2610 & 1.0 & 0.0053 & .. & .. & & \\
\hline 2385 & 1.3 & 0.0037 & $\therefore$ & 0 & 1.0 & 0.55 \\
\hline 2000 & 2.1 & 0.0018 & $=0$ & $\ldots$ & 1.7 & 0.27 \\
\hline 1600 & 4.8 & 0.00075 & $\ldots$ & $\ldots$ & 3.7 & 0.11 \\
\hline 1300 & 12 & 0.00033 & .. & & 9.1 & 0.049 \\
\hline 1000 & 51 & 0.00011 & 30 & 0.65 & 38 & 0.017 \\
\hline 800 & 240 & 0.00005 & 140 & 0.26 & 180 & 0.007 \\
\hline 700 & 750 & 0.00003 & 440 & 0.15 & 520 & 0.004 \\
\hline
\end{tabular}

Acknowledgments. I thank H. A. Weaver for the comet's HST digital images. The research described in this paper was carried out by the Jet Propulsion Laboratory, California Institute of Technology, under contract with the National Aeronautics and Space Administration and was supported in part through Grants GO-5021 and GO-5624 from the Space Telescope Science Institute, which is operated by the Association of Universities for Research in Astronomy, Inc.

\section{References}

Borovička, J. \& Spurný, P. 1995, Icarus, submitted

Boslough, M. B., Crawford, D. A., Robinson, A. C. \& Trucano, T. G. 1994, GRL, 21, 1555

Boslough, M. B., Crawford, D. A., Trucano, T. G. \& Robinson, A. C. 1995, GRL, 22, 1821

Bronshten, V. A. 1983, Physics of Meteoric Phenomena. Dordrecht: Reidel, 52

Carlson, R. W. et al. 1995, GRL, 22, 1557

Chapman, C. R. et al. 1995, GRL, 22, 1561

Chodas, P. W. 1995, personal communication

Graham, J. R., de Pater, I., Jernigan, J. G., Liu, M. C. \& Brown, M. E. 1995, Science, 267, 1320

Hammel, H. B. et al. 1995, Science, 267, 1288

Kompaneets, A. S. 1960, Sov. Phys. Dokl., 5, 46

McCrosky, R. E., Posen, A., Schwartz, G. \& Shao, C.-Y. 1971, JGR, 76, 4090

Nicholson, P. D. et al. 1995a, GRL, 22, 1613

Nicholson, P. D. et al. 1995b, GRL, 22, 1617

Sekanina, Z. 1983, AJ, 88, 1382; errata: AJ, 89, 185

Sekanina, Z. 1995, in European Shoemaker-Levy 9/Jupiter Workshop, R. M. West \& H. Boehnhardt, Garching bei München: ESO, 29

Watanabe, J.-i. et al. 1995, PASJ, 47, L21 